BNL-113492-2017-JA

\title{
Self-organized architectures from assorted DNA-framed nanoparticles
}

\author{
Wenyan Liu, Jonathan Halverson, Ye Tian, \\ Alexei V. Tkachenko and Oleg Gang
}

Submitted to Nature Chemistry

June 2016

Center for Functional Nanomaterials

Brookhaven National Laboratory

\author{
U.S. Department of Energy \\ USDOE Office of Science (SC), \\ Basic Energy Sciences (SC-22)
}

Notice: This manuscript has been authored by employees of Brookhaven Science Associates, LLC under Contract No. DE- SC0012704 with the U.S. Department of Energy. The publisher by accepting the manuscript for publication acknowledges that the United States Government retains a non-exclusive, paid-up, irrevocable, world-wide license to publish or reproduce the published form of this manuscript, or allow others to do so, for United States Government purposes. 


\section{DISCLAIMER}

This report was prepared as an account of work sponsored by an agency of the United States Government. Neither the United States Government nor any agency thereof, nor any of their employees, nor any of their contractors, subcontractors, or their employees, makes any warranty, express or implied, or assumes any legal liability or responsibility for the accuracy, completeness, or any third party's use or the results of such use of any information, apparatus, product, or process disclosed, or represents that its use would not infringe privately owned rights. Reference herein to any specific commercial product, process, or service by trade name, trademark, manufacturer, or otherwise, does not necessarily constitute or imply its endorsement, recommendation, or favoring by the United States Government or any agency thereof or its contractors or subcontractors. The views and opinions of authors expressed herein do not necessarily state or reflect those of the United States Government or any agency thereof. 


\title{
Self-organized architectures from assorted DNA-framed nanoparticles
}

\author{
Wenyan Liu, Jonathan Halverson, Ye Tian, Alexei V. Tkachenko and Oleg Gang ${ }^{\star}$
}

\begin{abstract}
The science of self-assembly has undergone a radical shift from asking questions about why individual components selforganize into ordered structures, to manipulating the resultant order. However, the quest for far-reaching nanomanufacturing requires addressing an even more challenging question: how to form nanoparticle (NP) structures with designed architectures without explicitly prescribing particle positions. Here we report an assembly concept in which building instructions are embedded into NPs via DNA frames. The integration of NPs and DNA origami frames enables the fabrication of NPs with designed anisotropic and selective interactions. Using a pre-defined set of different DNA-framed NPs, we show it is possible to design diverse planar architectures, which include periodic structures and shaped mesoobjects that spontaneously emerge on mixing of the different topological types of NP. Even objects of non-trivial shapes, such as a nanoscale model of Leonardo da Vinci's Vitruvian Man, can be self-assembled successfully.
\end{abstract}

T he act of manufacturing, even for the simplest of objects, is quite intricate. At its core, manufacture is the process of translating from an imaginary representation to a materialized realization. Traditional manufacturing is deterministic: a blueprint carries detailed information about positioning of the structural elements and the construction process. In contrast, nature's manufacturing of bio-organisms adopts a drastically different principle. Instead of being thoroughly explicit about the positions of all the elements, living systems are built via complex 'instructions' embedded in a system itself; such an approach makes living matter highly information efficient. For example, a human genome fits on two compact disks, a smaller data-storage requirement than modern computer operating systems. It is tempting to employ some of nature's principles to create the desired structures without prescribing the coordinates of each element in a system.

From this perspective, self-assembly can be viewed as a way to generate elaborate structures using minimal information; the information is 'encoded' in the system's components themselves. The past few decades of colloid self-assembly research have demonstrated that a wide variety of periodic and quasiperiodic organizations can be generated by tuning the shapes ${ }^{1-3}$ and interaction potentials of particles ${ }^{4-9}$; also, out-of-equilibrium ${ }^{10}$ and seeding ${ }^{11}$ approaches were considered to control the phase formations. Recently, directional binding ${ }^{12-15}$ has been exploited as a powerful means to control the formation of clusters ${ }^{16-20}$ and lattices ${ }^{21-23}$; for example, molecular analogues were formed from colloids with different valences ${ }^{16}$. Also recently, the formation of diamond-type lattices was demonstrated utilizing tetravalent binding motifs ${ }^{24}$, and a variety of designed lattices were shown using shaped DNA frames as complex assembly linkers ${ }^{25}$. However, as we strive to create materials with the desired functions, it is essential to find strategies for the self-assembly of arbitrarily designed architectures. In principle, adopting nature's approach, the instructions can be embedded into the building blocks. For instance, multitype anisotropic affinities ${ }^{26-29}$ were considered theoretically for creating structures with a modest complexity and high information content, and different types of assembled structures can be retrieved from the set of diverse building blocks ${ }^{30}$. In this work, we demonstrate experimentally a broadly applicable approach to creating prescribed planar architectures, both periodic and arbitrarily shaped, from a set of heterogeneous nanoparticle (NP) modules that are able to bind anisotropically and selectively. The block unifies a DNA construct and a NP in a highly tailorable module, which combines DNA programmability with NP functionality. Using the demonstrated strategy, broad classes of designed nanoscale systems, materials and devices can be formed in a self-assembled manner.

From an experimental point of view, two key challenges for this approach are the reliable large-scale fabrication of particles with the desired binding characteristics and the development of efficient lowerror assembly schemes. Particles with well-defined anisotropic interactions, so-called patchy particles ${ }^{31}$, are of broad interest for the assembly of clusters ${ }^{16,32,33}$ and large-scale organizations $s^{22,31,34,35}$. There are, however, only a few examples of their implementation at the nanoscale level ${ }^{36-38}$. Although controlling anisotropic connectivity is a significant challenge, many biological species (for example, viruses and multimeric proteins) and artificial DNA constructs (for example, tiled DNA motifs ${ }^{39-44}$, DNA origami ${ }^{45,46}$ and lego-blocks ${ }^{47}$ ) exhibit remarkable precision in this regard. The DNA nanotechnology has progressed to the level of creating sophisticated structures, yet, in the world of particles, approaches to the creation of NPs with designed binding properties are still limited. The approach demonstrated here effectively bridges particles and complex DNA nanostructures in the modules with highly adaptable interactions. Consequently, the desired architectures are formed in our approach via the stochastic co-assembly of different module types, as we demonstrate below.

\section{Results and discussion}

To implement the concept of anisotropic selective building blocks experimentally, we created modules from two-dimensional (2D) rigid DNA frames and gold nanoparticles (AuNPs) (Fig. 1a). These blocks are able to link between themselves in a planar manner via fourfold DNA-encoded interactions, as determined by the square-like DNA frame and the specificity encoded into the edges of the frames. We refer to the distinct interaction sites as having different 'colour' and therefore call these nano-objects polychromatic nanoparticles (pxNPs). These nano-objects possess both anisotropic binding properties and distinctive affinities provided 


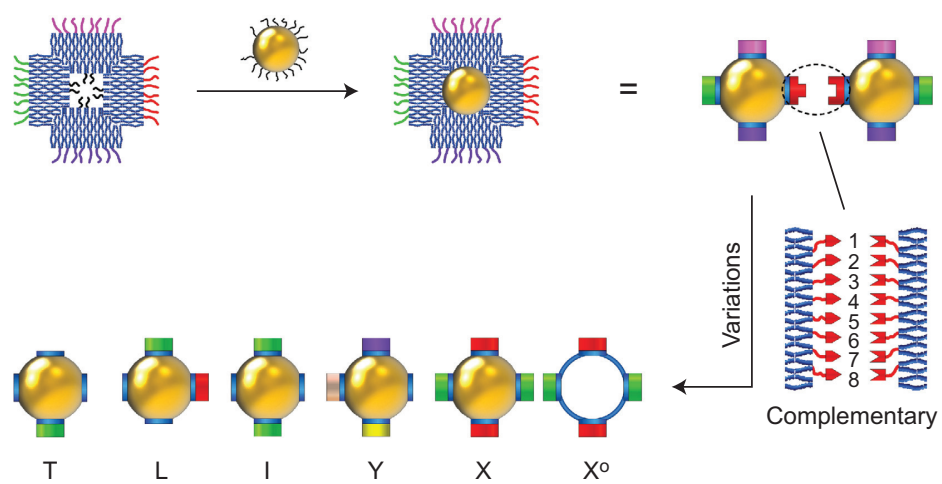

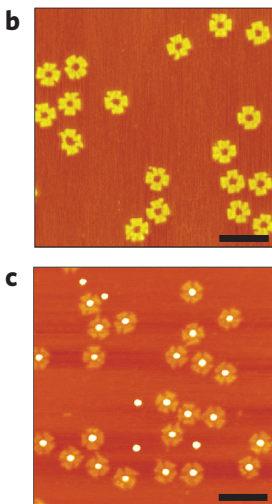

Figure 1 | Schematic diagram of nanoscale modules that incorporate NPs and DNA origami frames into $\mathrm{p} \chi \mathrm{NPs}$. $a$, Top row: schematic representation of the $\mathrm{p} \chi \mathrm{NP}$ assembly procedure. First, a long single-stranded circular M13 scaffold is folded by staple strands to create a DNA origami frame that contains capture strands inside the square window. Then, a DNA-functionalized AuNP binds inside the square window through complementary-strand hybridization to form the 2D pxNP. The different coloured strands on the outer edges of the structure represent the different coloured patches ( $\chi$ patches). The simplified drawing of the binding between the two pxNPs, as determined by the hybridization of eight distinctive DNA strands, is shown to the far right. Bottom row: the topological classes of the DNA frame-NP modules. $X^{\circ}$ is similar to an X-class particle, but does not incorporate a gold NP within the frame. $\mathbf{b}$, AFM image of DNA origami frames. c, AFM image of 2D pXNPs. Scale bars, $200 \mathrm{~nm}$.

by the 'coloured' edges ( $\chi$ patches, from $\chi \rho \tilde{\omega} \mu \alpha$ (chroma)). Thus, pXNPs can be viewed as generalized patchy particles with selective and fully prescribed anisotropic interactions. We demonstrate that diverse morphologies, such as linear chains, 2D lattices, simple shapes and even an arbitrarily designed object, can be created on demand from a library or 'alphabet' of these pxNP modules. We use a combination of atomic force microscopy (AFM), in situ $\mathrm{X}$-ray scattering and theoretical/numerical analysis to provide insight into this self-assembly approach.

Figure 1a illustrates schematically our assembly procedure for creating pXNPs with the help of 2D DNA origami frames. We employed a single-layer cross-shaped DNA origami tile $(\sim 100 \times 100 \mathrm{~nm})$ with a square window $(\sim 30 \times 30 \mathrm{~nm})$ in the centre as the basic frame unit (see Supplementary Fig. 1 and Supplementary Section 5 for the design details). This DNA frame acts as a particle's 'belt': eight internal 30-base single-stranded DNA (ssDNA) chains provide coupling to the complementary DNA on the particle (gold, $10 \mathrm{~nm}$ diameter) surface, whereas the outer sides of the square frame contain ssDNA for frame-frame recognition. The four outer sides of the DNA origami frame can be encoded individually by sets of ssDNAs to act as four $\chi$ patches, as discussed below. The framed NP is quasi-2D because the four connectors can form only in-plane bonds.

We produced the framed NPs by mixing and annealing the freshly prepared DNA frames and the NP-DNA conjugates (see Methods for details). The formations of the DNA frames and the framed NPs were verified by AFM. To enable the analysis of individual species, four-base poly-T tails were added to the corresponding staple strands located at the outer edges of the frame to prevent frame-frame stacking. As can be seen in Fig. 1b,c, respectively, the origami frames were formed as designed in high yield (>99\%) with a highly homogeneous shape and the NPs were embedded in frames via DNA hybridization with a nearly perfect filling yield. To allow the framed NP functions as a desired type of pxNP, a set of rationally designed sticky ends, which serves as a $\chi$ patch, was introduced into one or more edges of the frame by extending selected staple strands. Each $\chi$ patch contains eight different fivebase sticky ends that have distinct sequences arranged in a prescribed order. This restricts the assembly between two frames with complementary sequences, which forces the frames to align exactly and prevents uncontrolled lateral shifting. Thus, the combination of anisotropic and DNA-encoded 'chromatic' properties of bonds permits the creation of a programmable module, $\mathrm{pXNP}$, as shown at the top right of Fig. 1a.
The major classes of polychromatic particles in our study are illustrated in the bottom row of Fig. 1a, and they are determined by the topology of their connections. We assign letters to these different topological classes of pxNPs: $\mathrm{T}$ (one-connector terminal particle), L (two-connector particles with a $90^{\circ}$ valence angle), I (two-connector particles with a $180^{\circ}$ valence angle), Y (threeconnector particles) and $\mathrm{X}$ (four-connector particles). These major modules can be diversified further by changing the DNA encoding. Essentially, this set of modular building blocks represents a particle 'alphabet', with each pxNP type being a 'letter'. The combination of topological classes with specific 'colouring' of the bonds provides a fully customizable set of these modules (see Supplementary Table 1 for the full list of 21 pxNPs).

We first demonstrate the assembly of targeted structures ('words'): chain-like 1D arrays and simple shapes using the four module classes, T, L, I and X (Fig. 2a). For example, the assembly of a homogeneous square-shaped cluster requires two kinds of L-class pxNPs, $\mathrm{L}_{12}$ and $\mathrm{L}_{\overline{2} \overline{1}}$ (Fig. $2 \mathrm{a}$, route $\mathrm{A}$ ). In contrast, the assembly of the cross-shaped cluster requires two different topologies of pXNPs, one-connector terminal particles $\left(\mathrm{T}_{1}, \mathrm{~T}_{2}\right)$ and a four-connector central particle, $\mathrm{X}_{\overline{1} \overline{2}}$ (Fig. $2 \mathrm{a}$, route $\mathrm{B}$ ). To construct these nanoclusters, a one-pot annealing procedure was adopted, in which DNA-coated NPs and the pre-assembled DNA origami frames were mixed together to form, initially, pxNPs, which subsequently self-assembled into the targeted morphologies via the recognition of $\chi$ patches (see Methods for details, and Supplementary Section 2 for an analysis of the melting temperatures).

The morphology of the resultant nanostructures was visualized directly with AFM. Figure 2b,d show representative AFM images of the assembled clusters (also see Supplementary Fig. 2). The structures are consistent with the prescribed cluster types, squareand cross-like, respectively. Partially assembled clusters show the formation of incomplete but correct morphologies. The yield analysis from the AFM images reveals rather high mass fractions of successfully assembled target morphologies: the histograms (Fig. 2c,e) demonstrate that the assembly efficiencies of the square-shaped cluster and the cross-shaped cluster are about 54 and 57\%, respectively (based on statistical analyses of 3,481 and 2,462 particles, respectively). The formation yields obtained from AFM are in agreement with estimations from gel electrophoresis (see Supplementary Section 4). By incorporating particles of different sizes in complementary frames, we verified that a two-step annealing protocol (the formation of framed particles first and 
a
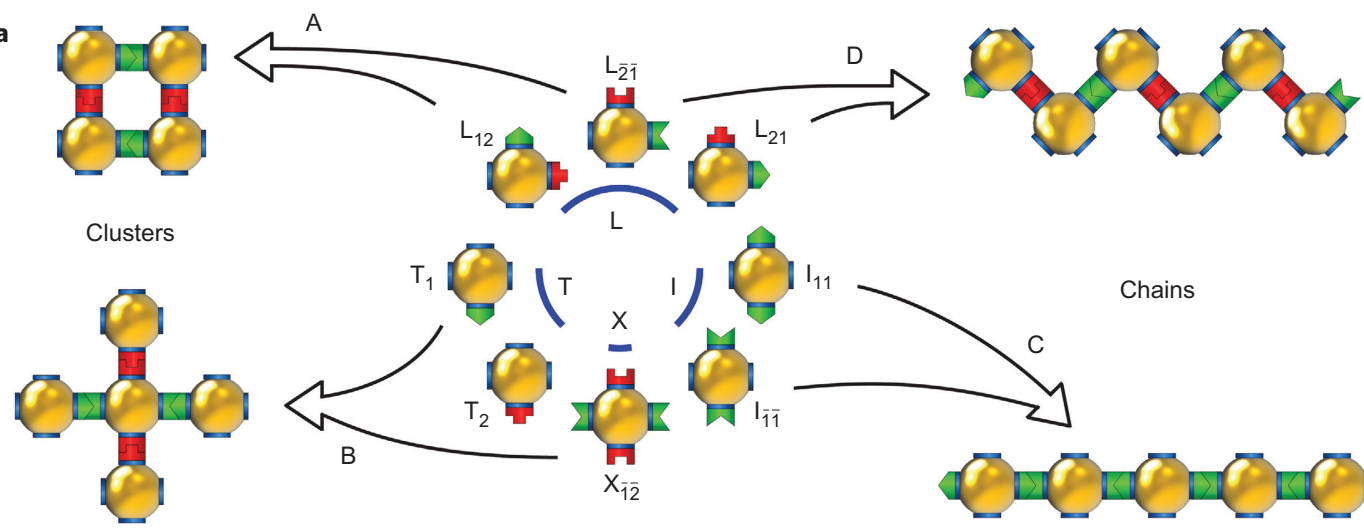

Chains
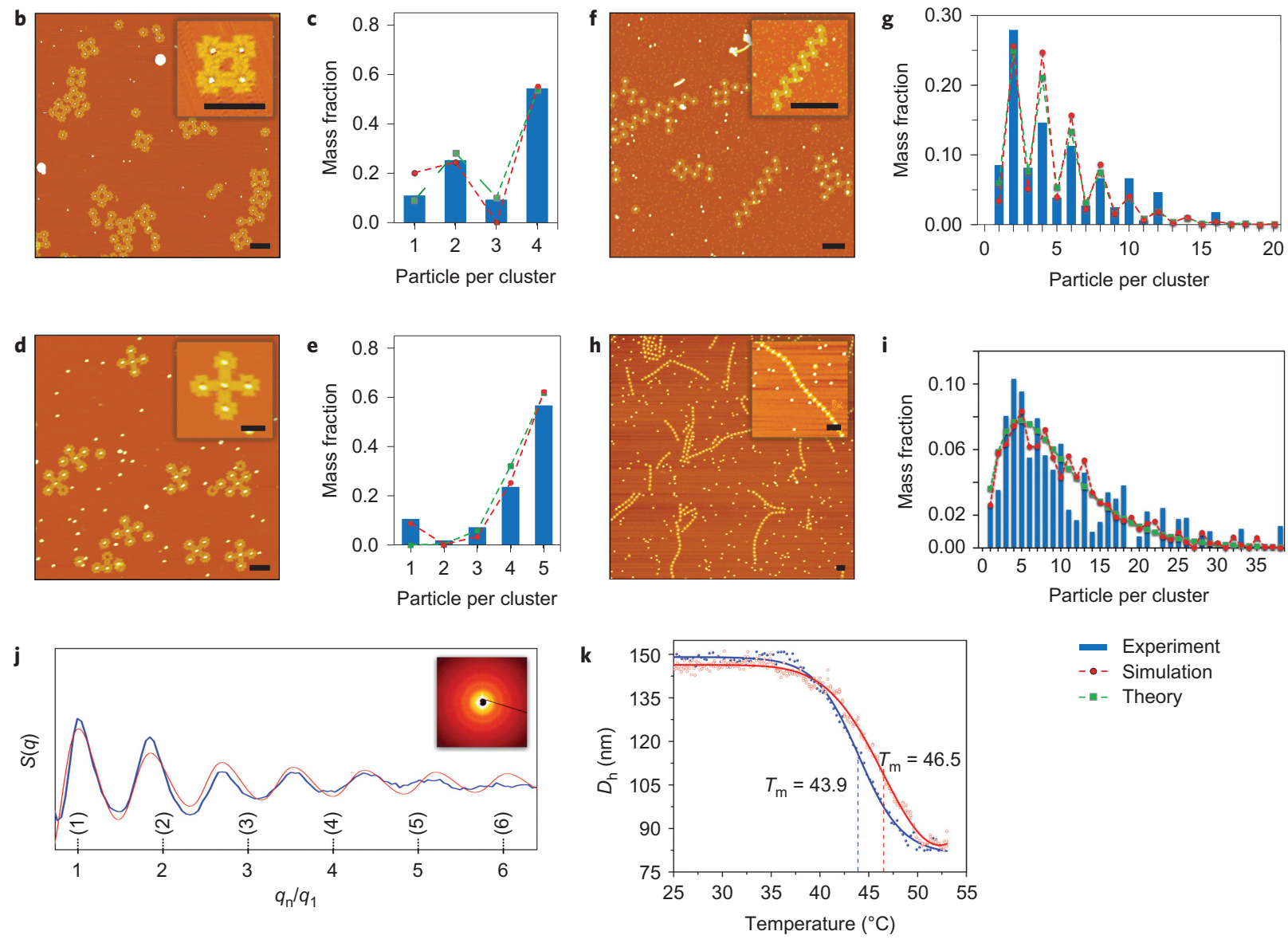

Figure 2 | Nanoclusters and 1D arrays assembled from the pXNPs. a, Schematic drawing of the assembly processes. Route A, square-shaped cluster; route $B$, cross-shaped cluster; route $C$, linear chain and route D, zigzag chain. b-i, AFM images of the square-shaped (b) and cross-shaped (d) clusters, and the zigzag (f) and linear (h) 1D arrays. A comparison of the linked NP mass fractions, as observed by AFM, evaluated by theory (green dashed lines) and obtained from the Brownian dynamics simulation (red dashed lines), is shown for the square-shaped clusters (c), the cross-shaped clusters (e), the zigzag (g) and linear (i) chain systems. j, Structure factor $S(q)$ for linear $1 D$ arrays as a function of $q_{n} / q_{1}$. The blue and red curves correspond to the experimental result and fitting, respectively, and the dotted lines (black) are the calculated positions of the diffraction peaks for the linear 1D arrays. The inset is a representative 2D SAXS pattern for the linear chain. $\mathbf{k}$, Melting-curve analysis of the $1 \mathrm{D}$ array, presented as hydrodynamic diameter $\left(D_{\mathrm{h}}\right)$, measured by DLS as a function of temperature. Blue, binding interaction along the horizontal direction; red, binding interaction along the vertical direction. Bottom right, the legend for c,e,g and i. Scale bars, $200 \mathrm{~nm}$.

then the assembly of clusters) also results in a successful structure formation (see Supplementary Fig. 9). Moreover, this demonstrates that heteroparticle systems can be formed using the same approach.

The observed histograms are in good agreement with the theoretical equilibrium distributions, as shown in Fig. 2c,e. In particular, for the case of a cross-shaped cluster, the mass fractions of $n$ particle products follow the binomial distribution $\left(M_{n}=n C_{4}^{n-1}(1-p)^{5-n} p^{n-1}\right) / 5$ (valid for $1<n<6)$ for the experimental molar 1:4 ratio of $\mathrm{X}$-class to
T-class particles. Here $p$, the probability of formation of an individual chromatic bond, can be related to the binding free energy, $\Delta G$, and to the concentration, $C$, of particles (of each type) through the standard relationship for an equilibrium constant in a reversible binding:

$$
p /(1-p)^{2}=C \mathrm{e}^{(-\Delta G / k T)}
$$




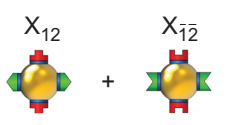

II

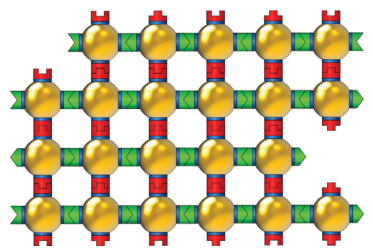

b
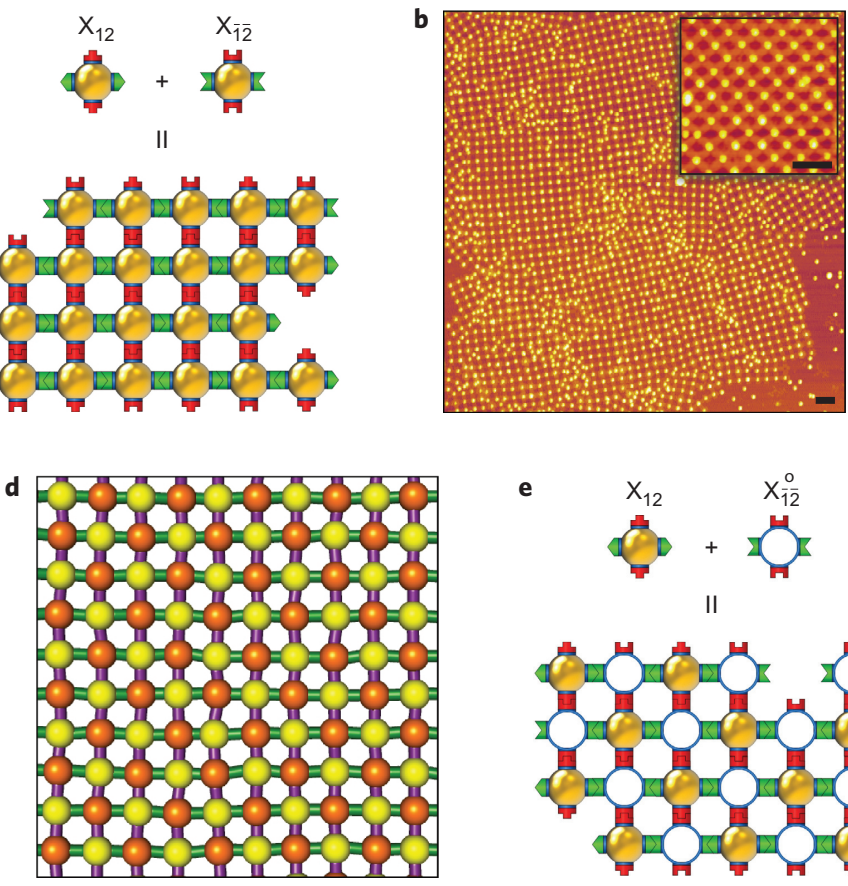

e
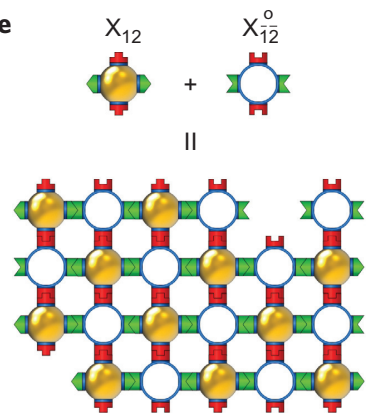
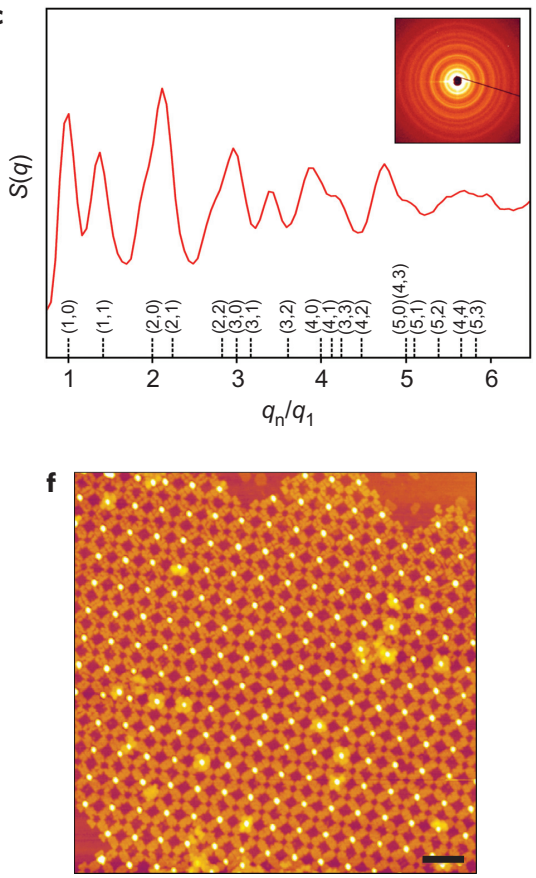

Figure 3 | Assembly of 2D square NP arrays. a-d, 2D NP arrays assembled from $X_{12}$ and $X_{\overline{1} 2} P \chi N P s$. a, Schematic drawing of the structure formation process. b, AFM image of 2D arrays. The interparticle distance is $\sim 95 \mathrm{~nm}$. Inset: a zoomed-in image of a 2D array. c, SAXS-obtained structure factor, S( $q$ ). The solid curve (red) is the experimental result, and the dotted lines (black) are the calculated diffraction positions for the 2D lattice. Inset: representative 2D SAXS pattern of the 2D arrays. d, Snapshot of the 2D array obtained by Brownian dynamics simulation, as discussed in the Supplementary Information. e,f, $2 \mathrm{D}$ NP arrays assembled from $\mathrm{X}_{12}$ and $\mathrm{X}_{\overline{1} \mathrm{2}}^{\circ} \mathrm{p} \chi N P \mathrm{~s}$. e, Schematic drawing of the array formation process. $\mathbf{f}, \mathrm{AFM}$ image of $2 \mathrm{D}$ arrays. The interparticle distance is $\sim 190 \mathrm{~nm}$. Scale bars, $200 \mathrm{~nm}$.

For simplicity, we neglected the difference in binding free energy $(\Delta G)$ between the two bond types employed. However, this difference is crucial to understand the non-trivial product distribution for the square-shaped cluster, as we discuss below. Additionally, we performed Brownian dynamics simulations of chromatic directionally functionalized NPs (see Supplementary Section 8) ${ }^{26}$, and the results (red dashed lines in Fig. 2c,e) agree with experiment and analytical theory.

An appropriate mixing of $\mathrm{pxNPs}$ can also form linear arrays that mimic homo- and heteropolymers, using one and two kinds of bonds, respectively. The $1 \mathrm{D}$ chain (homopolymer) morphology can be formed when I class (bond angles of $180^{\circ}$ ) particles, $\mathrm{I}_{11}$ and $\mathrm{I}_{\overline{1} \overline{1}}$, are assembled at a ratio of $1: 1$ (Fig. $2 \mathrm{a}$, route $\mathrm{C}$ ). In this case, there is only one type of interparticle interaction (11) between these pXNPs. AFM measurements demonstrate that string-like particle arrays of various lengths are formed (Fig. $2 \mathrm{~h}$ and Supplementary Fig. 3a), with an average interparticle distance $(D)$ of about $95 \mathrm{~nm}$. The longest 1D chain was found to be 38 particles long, with a relatively straight conformation on the substrate, but the majority of the chains are 5-15 particles long (Fig. 2i). By probing these $1 \mathrm{D}$-assemblies in solution with in situ synchrotron-based small-angle X-ray scattering $(\mathrm{SAXS})^{5}$, as shown in Fig. $2 \mathrm{j}$, we confirmed that chains were formed in the bulk with an average interparticle distance of $\sim 89 \mathrm{~nm}$ (see Methods for details), close to the in-air AFM results.

The histogram of observed chain lengths exhibits pronounced exponential behaviour (Fig. 2i). A theoretical fit to this distribution can be obtained within the same equilibrium model as that used for the finite clusters above. Accordingly, the corresponding mass fraction is expressed as $M_{n}=n(1-p)^{2} p^{n-1}$, which matches the data for $p=0.81$. This equilibrium distribution is formally equivalent to the classic result of the (irreversible) step-growth polymerization theory originally proposed by Flory ${ }^{48}$; however, the average chain length in our case is determined by the bond strength rather than by kinetics.
In addition to linear chains, we programmed the self-assembly of heteropolymers with a 'zigzag' morphology (Fig. 2a, route D). These chains are formed by the association between $\mathrm{L}$ class $\left(90^{\circ}\right.$ bond angle) particles, $\mathrm{L}_{21}$ and $\mathrm{L}_{\overline{2} \overline{1}}$, through two types of bond interactions ( $1 \overline{1}$ and $2 \overline{2}$ ). For $1: 1$ mixtures of $L_{21}$ and $L_{\overline{2} \overline{1}}$ pXNPs, zigzag chains match the intended design (see Fig. $2 \mathrm{f}$ and Supplementary Fig. $3 b$ ). The average length of the assembled zigzags is significantly shorter than that for the straight linear chains. Furthermore, a strong oddeven effect in the chain-length distribution is observed (Fig. 2g). This effect is, indeed, expected for the equilibrium distribution if the two bond types have somewhat different strengths. Using our theoretical model (see Supplementary Section 7), the resulting mass fraction can be presented as:

$$
M_{n}=\mathrm{e}^{\beta \epsilon} n p^{n}\left\{\begin{array}{cc}
1 & n-\text { odd } \\
\frac{1}{2}\left(X+\frac{1}{X}\right) & n-\text { even }
\end{array}\right.
$$

Here $\epsilon=\left(\left(\Delta G_{1}+\Delta G_{2} / 2\right)-k T \log C\right.$, parameter $p$ is given by equation (S4) in Supplementary Section 7. The strength of the odd-even effect is determined by the parameter $X=\exp \left(\left(\Delta G_{1}-\Delta G_{2}\right) / 2 k T\right)$. This model explains the enhanced population of 'even' zigzag chains compared with the 'odd' ones, as they contain unequal numbers of the two energetically different bonds. This conclusion is further supported by comparing the melting behaviour of systems with the two distinct bond types using dynamic light scattering (DLS (Fig. 2k and Supplementary Section 2)), and a difference in bond strengths, $\sim k T$, was observed. The very same effect is responsible for the non-trivial product distribution of the square-shaped clusters (Fig. 2c). Although the complete square was, indeed, the dominant structure, the presence of dimers is another manifestation of the above odd-even effect. In fact, this distribution is well described by equation (2) for $n=1,2$ and 3 , and a high fraction of the 
a

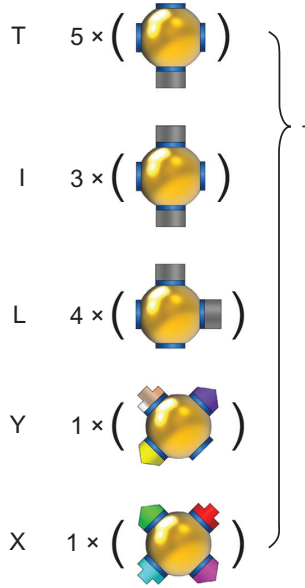

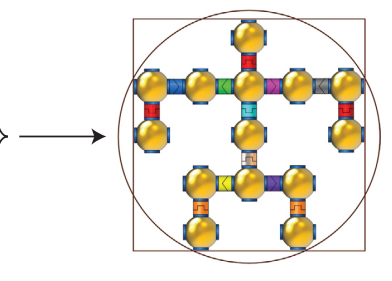

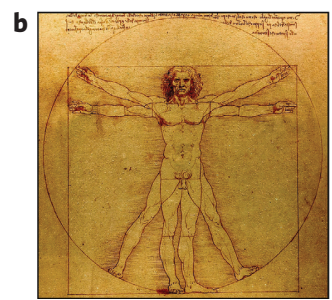

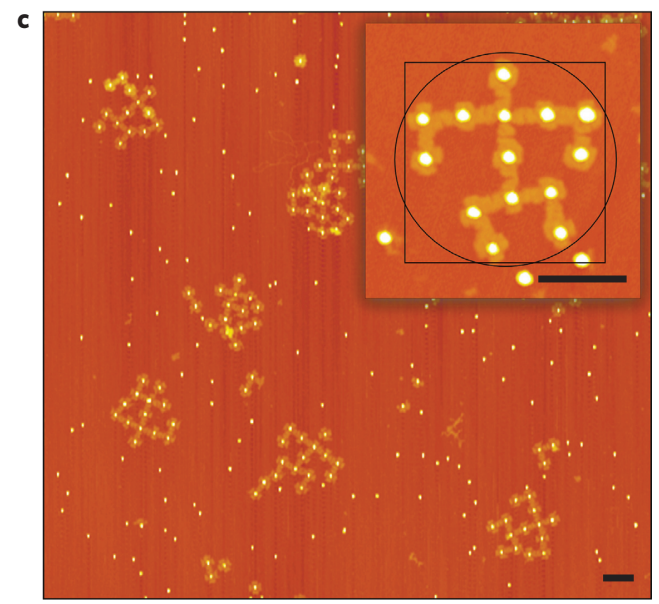

Figure 4 | Designed architecture: nanoscale analogue of Leonardo da Vinci's Vitruvian Man, assembled from multiple types of p $\chi$ NPs. a, Schematic drawing of the formation process. This nanocluster is composed of 14 p $\chi N P s$ (11 different kinds of five types (see the Supplementary Information)). For simplicity, only five topologically different $p \chi N P$ types $(T, I, L, Y, X)$ are shown here, and the grey patch represents a generic colour encoding that can be converted into the desired $\chi$-patch type. b, Leonardo da Vinci's Vitruvian Man. c, Representative AFM image of the assembled nanoscale Vitruvian Man clusters. Inset: a high-magnification view of a well-assembled structure of the designed nanoscale Vitruvian Man. Scale bars, 200 nm.

complete square cluster $(n=4)$ results from the extra bond for the closed-loop topology.

The stochastic character of our modular self-assembly approach permits the manipulation of assembled structures by adjusting the stoichiometric ratio of the involved pXNPs. To explore this effect, we performed two sets of experiments, as illustrated in Supplementary Fig. 4 . The previously described particles $\left(\mathrm{I}_{11} \& \mathrm{I}_{\overline{1} \overline{1}}\right)$, used to form linear chains (Fig. $2 a$, route $C$ ), were mixed at different stoichiometric ratios. Both the experimental and simulation data suggest the formation of chains with odd-even effects and much shorter lengths than for a 1:1 ratio. Remarkably, the effect is opposite to that of the zigzag case: 'even' chains are suppressed with respect to the 'odd' ones, consistent with the theoretical prediction. This demonstrates that our strategy can tune and control structural order even in the presence of stochastic assembly and despite the distribution of assembled states.

The X-class particles further broaden the range of possible structures that can be formed. The four-patch pxNPs $\left(\mathrm{X}_{12}\right.$ and $\left.\mathrm{X}_{\overline{1} \overline{2}}\right)$ have $90^{\circ}$ bond angles, which encode a $2 \mathrm{D}$ square-lattice symmetry (Fig. 3a). AFM images demonstrate the successful formation of the intended lattice, with exceptional micrometre domain sizes (Fig. 3b and Supplementary Fig. 5) and a high spatial fidelity (Fig. 3b, inset). The well-defined SAXS pattern confirms the lattice formation at $\sim 45^{\circ} \mathrm{C}$ (see Supplementary Fig. 6), which exhibits a remarkable degree of long-range order even in bulk solution, as indicated by more than 20 orders of Bragg peaks (Fig. 3c). The normalized peak positions, located at $q_{n} / q_{1}=1: \sqrt{2}: 2: \sqrt{5}: \sqrt{8} \ldots\left(q_{1}=0.0075 \AA^{-1}\right)$, unambiguously identify a $2 \mathrm{D}$ square-lattice structure with a lattice constant of $83.8 \mathrm{~nm}$. The excellent correspondence with the theoretical peak positions of the low-index diffraction peaks (dotted lines in Fig. 3c) implies a high degree of order, whereas some deviation for the higherorder peaks suggests that the free-floating $2 \mathrm{D} \mathrm{NP}$ sheets have a certain degree of flexibility. To demonstrate further the generality of this approach, we assembled a more-complex 2D lattice by combining hollow and NP-filled pxNPs, $\mathrm{X}_{12}$ and $\mathrm{X}_{\overline{1}}^{\mathrm{o}}$, respectively (Fig. 3e). The resulting lattice (Fig. 3f) exhibits excellent order and doubles the interparticle distance in comparison with the case shown in Fig. 3a.

Finally, we demonstrate the versatility of the presented assembly strategy for the creation of an arbitrary targeted architecture by designing an anthropomorphically shaped nanocluster (Fig. 4a). Its major proportion (height/width) is similar to the classic
Leonardo da Vinci's Vitruvian Man (Fig. 4b). Although the symmetric finite-size clusters and low-dimensional arrays discussed above can be encoded by binary mixtures of polychromatic particles, the nano Vitruvian Man requires a larger set of building blocks. Therefore, this experiment also allows tests of the scalability of the presented assembly approach. The stochastic assembly of such an architecture is significantly more intricate because it requires the use of all the proposed topological classes (Fig. 1). The composition of the mixture was chosen according to the stoichiometric ratio in the target structure. Each nanoscale Vitruvian Man is composed of 14 pXNPs that represent 11 different types within the five topological classes of modules: $\mathrm{T}$ (one $\chi$ patch), $\mathrm{L}$ (two $\chi$ patches, $90^{\circ}$ bond angle), I (two $\chi$ patches, $180^{\circ}$ bond angle), $Y$ (three $\chi$ patches, $90^{\circ}$ bond angle) and $X$ (four $\chi$ patches, $90^{\circ}$ bond angle), as illustrated in Fig. $4 \mathrm{a}$ (see Supplementary Fig. 7 for the details). We observed the formation of high-fidelity architectures (Fig. 4c) that exactly matched our design of Vitruvian Man, which further demonstrates the enormous potential of the proposed assembly concept.

\section{Conclusion}

We have demonstrated a simple yet versatile strategy for the predictable assembly of planar structures, including periodic arrays, simple shapes and complex meso-architectures, using nanoscale modules with anisotropic and selective bindings. Our building modules integrate NPs and DNA frames into unified and highly tailorable objects: the DNA-framed particles carry both material function through the NP and precise binding characteristics through DNA encoding. Furthermore, particles of different types and sizes can be utilized to build hetero-assemblies for potential applications in catalysis and in optical and magnetic materials, for which the desired separations can be achieved by designing a frame and the particle location within it. As the proposed approach permits the incorporation of different particle types and the use of specifically designed DNA frames with the desired symmetries of interaction, a large diversity of architectures can be created on demand. For example, the use plasmonic particles with the desired resonance properties will permit us to create plasmonic filters or coatings, and by arranging particles in a directional manner the rotation of light can be manipulated. Our detailed structural studies reveal the excellent correspondence between the targeted structures and their experimental self-assembled realizations. Importantly, our theoretical studies show that the assembly process, population and morphological details of the 
formed architectures can all be predicted quantitatively and can be manipulated based on system design. Although modules based on the four-fold symmetry are demonstrated here, the methodology can certainly be extended to other symmetries, to their combination and, potentially, to the design and fabrication of $3 \mathrm{D}$ architectures. Moreover, our approach allows for the incorporation of a broad variety of NP functional types ${ }^{49,50}$. Thus, it opens up tremendous possibilities for creating fully designed functional materials and devices using the demonstrated rational-design self-assembly approach.

\section{Methods}

Design, synthesis and purification of DNA. The sequences of sticky ends were designed using the program SEQUIN ${ }^{51}$. The single-stranded M13mp18 DNA genome was purchased from Bayou Biolabs. All unmodified staple strands were purchased from Integrated DNA Technologies, Inc. in RNase-free water at $100 \mu \mathrm{M}$ and used without further purification. All sticky-end-containing staple strands, anchor-containing staple strands and 3'-thiolated DNA strands were also purchased from IDTDNA and purified using denaturing PAGE.

Preparation of gold NP-DNA conjugates. The 3'-thiolated oligonucleotides were reduced for two hours by tris-(2-carboxyethyl) phosphine (TCEP) in water with a TCEP:DNA ratio of 100:1, and subsequently purified using size-exclusion columns (G-25, GE Healthcare) to remove small molecules. Then, the thiolated oligonucleotides were added into the aqueous solution that contained $10 \mathrm{~nm}$ gold NPs (Ted Pella, Inc.) at a DNA/NP ratio of 300:1. After two hours, the solution was brought to $10 \mathrm{mM}$ in phosphate ( $\mathrm{pH} 7.4$ ) and allowed to age for two hours. In the subsequent stepwise salt-ageing process, a $2 \mathrm{M} \mathrm{NaCl}$ solution was gradually added to the colloid solution to reach a final $\mathrm{NaCl}$ concentration of $0.3 \mathrm{M}$ (six steps with a one hour waiting period between each addition), and the solution was allowed to age for another 12 hours. To remove unbound DNA, the solution was centrifuged and the supernatant removed using a pipette. The DNA-gold NP conjugates were washed with a $0.1 \mathrm{M} \mathrm{NaCl}, 10 \mathrm{mM}$ phosphate buffer and recentrifuged four times. Finally, the functionalized NPs were resuspended in a freshly prepared $0.1 \mathrm{M} \mathrm{NaCl}, 10 \mathrm{mM}$ phosphate buffer, and the concentration was estimated from the optical absorbance at $520 \mathrm{~nm}$.

\section{Structure formation by thermal annealing}

Self-assembly of DNA origami frame. The mixture of staple strands, sticky-ended strands, anchor strands and M13mp18 DNA genome was brought to a volume of $100 \mu \mathrm{l}$ using $1 \times$ TAE buffer solution containing $40 \mathrm{mM}$ Tris- $\mathrm{HCl}, \mathrm{pH}$ 8.0, $20 \mathrm{mM}$ acetic acid, $2.5 \mathrm{mM}$ EDTA and $12.5 \mathrm{mM}$ magnesium acetate. The final concentration of the M13mp18 DNA genome in the solution was $20 \mathrm{nM}$, and the molar ratio between the long viral ssDNA, the unmodified staple strands, the stickyended strands and the anchor strands was 1:5:3:1. The sample was cooled from 90 to $16^{\circ} \mathrm{C}$ over the course of $\sim 12$ hours in a thermocycling machine (Eppendorf).

Self-assembly of $p \chi N P s$. After the DNA origami frames were annealed, the freshly prepared DNA origami frames and gold NP-DNA conjugates were mixed in a 1:2 molar ratio, and slowly annealed from $53{ }^{\circ} \mathrm{C}$ to room temperature for 12 hours to allow them to hybridize together to form the pXNPs.

Programmable self-assembly gold nanostructures from the pXNPs. The DNA-coated $10 \mathrm{~nm}$ gold NPs were mixed with the corresponding pre-assembled DNA origami frames in a ratio of 2:1, and slowly cooled over $\sim 60$ hours from 53 to $25^{\circ} \mathrm{C}$ in an incubator (Echotherm TM IN35).

AFM imaging. A $5 \mu$ sample was spotted on freshly cleaved mica and left to adsorb to the surface over two minutes. To remove salts, the mica surface was rinsed with Millipore water $(30 \mu \mathrm{l})$ three times. Then the mica was wicked dry and further blowdried with compressed air. All the samples were scanned in tapping-in-air mode on an Asylum MFP3D AFM (Asylum Research) with NSC14/Al BS (MikroMasch).

SAXS. SAXS was performed at the National Synchrotron Light Source (NSLS) X-9 beamline. The samples were measured in quartz capillary tubes. The scattering data were collected with a CCD (charge-coupled device) area detector, and then integrated into a 1D scattering curve $I(q)$ as a function of the scattering momentum transfer $q$, where $q=(4 \pi / \lambda) \sin (\theta / 2)$, with $\lambda$ and $\theta$ being the wavelength of incident $\mathrm{X}$-rays and the scattering angle, respectively. The structure factor $S(q)$ was obtained by dividing $I(q)$ by the particle-form factor $F(q)$ determined from a suspension of unaggregated free particles.

SAXS modelling of the linear NP arrays. The diffraction pattern was modelled by using the functional form $\sum_{n} R^{-n} \sin (n D q) / n D q$, where $n$ is an integer number and the factor $R^{-n}$ accounts for the reduced correlation with chain increase because of its flexibility and finite-size length; this yields $D \approx 89 \mathrm{~nm}$. Such flexibility of the chain structure and its in-liquid condition might be responsible for small interparticle distance differences, as observed by SAXS and AFM.
Analysis of the melting behaviour of the binding interactions. To verify further that the bonding is reversible on the time scale of the experiment and to estimate the difference in binding free energies between different bond types, we analysed the system's melting behaviour. We performed DLS measurements for two kinds of homopolymer-type systems that grow along the horizontal and vertical directions, respectively (two different bond types as defined above). As shown in

Supplementary Fig. 4, the reversibility is, indeed, confirmed because the heating and cooling curves closely match. In addition, the difference in binding interactions along the horizontal and vertical directions (blue and red curves, respectively, in Fig. $2 \mathrm{k}$, and Supplementary Section 2) resulted in a $\sim 3^{\circ} \mathrm{C}$ difference in melting temperature. By fitting the data with a standard melting curve, equation (1), we can determine entropy for each bond type, $S=-\partial G / \partial T$. From these data one can extract the difference in free energy for the two bonds using a linear extrapolation from melting points $T_{\mathrm{m} 1}$ and $T_{\mathrm{m} 2}$ to the experimental temperature $T$. Owing to a high sensitivity of fitting, this method can only reliably provide an order of magnitude estimate, $\Delta G=\Delta S_{1}\left(T_{\mathrm{m} 1}-T\right)-\Delta S_{2}\left(T_{\mathrm{m} 2}-T\right) \approx k T$. On the other hand, the value of this parameter required to explain the observed amplitude of the odd-even effect is $\Delta G \approx 4 k T$. Although the two results are generally consistent, a certain discrepancy may be attributed to the significantly different conditions at which the two types of experiments had to be performed.

Received 17 July 2015; accepted 28 April 2016; published online 13 June 2016

\section{References}

1. Damasceno, P. F., Engel, M. \& Glotzer, S. C. Predictive self-assembly of polyhedral into complex structures. Science 337, 453-457 (2012).

2. Agarwal, U. \& Escobedo, F. A. Mesophase behaviour of polyhedral particles. Nature Mater. 10, 230-235 (2011).

3. Whitelam, S., Tamblyn, I., Garrahan, J. P. \& Beton, P. H. Emergent rhombus tilings from molecular interactions with M-fold rotational symmetry. Phys. Rev. Lett. 114, 115702 (2015).

4. Jain, A., Errington, J. R. \& Truskett, T. M. Inverse design of simple pairwise interactions with low-coordinated 3D lattice ground states. Soft Matter $\mathbf{9}$, 3866-3870 (2013).

5. Nykypanchuk, D., Maye, M. M., van der Lelie, D. \& Gang, O. DNA-guided crystallization of colloidal nanoparticles. Nature 451, 549-552 (2008).

6. Park, S. Y. et al. DNA-programmable nanoparticle crystallization. Nature 451, 553-556 (2008).

7. Podsiadlo, P., Krylova, G. V., Demortiere, A. \& Shevchenko, E. V. Multicomponent periodic nanoparticle superlattices. J. Nanopart. Res. 13, 15-32 (2011).

8. Xu, L. et al. Nanoparticle assemblies: dimensional transformation of nanomaterials and scalability. Chem. Soc. Rev. 42, 3114-3126 (2013).

9. Talapin, D. V. et al. Quasicrystalline order in self-assembled binary nanoparticle superlattices. Nature 461, 964-967 (2009).

10. Whitelam, S., Schulman, R. \& Hedges, L. Self-assembly of multicomponent structures in and out of equilibrium. Phys. Rev. Lett. 109, 265506 (2012)

11. Barish, R. D., Schulman, R., Rothemund, P. W. K. \& Winfree, E. An informationbearing seed for nucleating algorithmic self-assembly. Proc. Natl Acad. Sci. USA 106, 6054-6059 (2009).

12. Romano, F. \& Sciortino, F. Two dimensional assembly of triblock Janus particles into crystal phases in the two bond per patch limit. Soft Matter 7, 5799-5804 (2011).

13. Smallenburg, F. \& Sciortino, F. Liquids more stable than crystals in particles with limited valence and flexible bonds. Nature Phys. 9, 554-558 (2013).

14. Yi, G. R., Pine, D. J. \& Sacanna, S. Recent progress on patchy colloids and their self-assembly. J. Phys. Condensed Matter 25, 193101 (2013).

15. van Ravensteijn, B. G. P., Kamp, M., van Blaaderen, A. \& Kegel, W. K. General route toward chemically anisotropic colloids. Chem. Mater. 25, 4348-4353 (2013).

16. Wang, Y. F. et al. Colloids with valence and specific directional bonding. Nature 491, 51-61 (2012).

17. Ye, X. et al. Competition of shape and interaction patchiness for self-assembling nanoplates. Nature Chem. 5, 466-473 (2013).

18. Klinkova, A., Therien-Aubin, H., Choueiri, R. M., Rubinstein, M. \& Kumacheva, E. Colloidal analogs of molecular chain stoppers. Proc. Natl Acad. Sci. USA 110, 18775-18779 (2013).

19. Lau, K. L., Hamblin, G. D. \& Sleiman, H. F. Gold nanoparticle 3D-DNA building blocks: high purity preparation and use for modular access to nanoparticle assemblies. Small 10, 660-666 (2014).

20. Sun, D. Z. et al. Heterogeneous nanoclusters assembled by PNA-templated double-stranded DNA. Nanoscale 4, 6722-6725 (2012).

21. Jones, M. R. et al. DNA-nanoparticle superlattices formed from anisotropic building blocks. Nature Mater. 9, 913-917 (2010).

22. Chen, Q., Bae, S. C. \& Granick, S. Directed self-assembly of a colloidal Kagome lattice. Nature 469, 381-384 (2011).

23. Knorowski, C. \& Travesset, A. Self-assembly and crystallization of hairy (f-star) and DNA-grafted nanocubes. J. Am. Chem. Soc. 136, 653-659 (2014). 
24. Liu, W. et al. Diamond family of nanoparticle superlattices. Science 351, 582-586 (2016).

25. Tian, Y., Wang, T., Zhang, Y., Li, H. \& Gang, O. Lattice engineering via nanoparticle-DNA frameworks. Nature Mater. 15, 654-661 (2016).

26. Halverson, J. D. \& Tkachenko, A. V. DNA-programmed mesoscopic architecture. Phys Rev. E 87, 062310 (2013).

27. Tkachenko, A. V. Theory of programmable hierarchic self-assembly. Phys. Rev. Lett. 106, 255501 (2011).

28. Murugan, A., Zou, J. \& Brenner, M. P. Undesired usage and the robust selfassembly of heterogeneous structures. Nature Commun. 6, 6203 (2015).

29. Jacobs, W. M., Reinhardt, A. \& Frenkel, D. Rational design of self-assembly pathways for complex multicomponent structures. Proc. Natl Acad. Sci. USA 112, 6313-6318 (2015).

30. Murugan, A., Zeravcic, Z., Brenner, M. P. \& Leibler, S. Multifarious assembly mixtures: systems allowing retrieval of diverse stored structures. Proc. Natl Acad. Sci. USA 112, 54-59, (2015).

31. Ruzicka, B. et al. Observation of empty liquids and equilibrium gels in a colloidal clay. Nature Mater. 10, 56-60 (2011).

32. Feng, L., Dreyfus, R., Sha, R. J., Seeman, N. C. \& Chaikin, P. M. DNA patchy particles. Adv. Mater. 25, 2779-2783 (2013).

33. Kraft, D. J. et al. Patchy polymer colloids with tunable anisotropy dimensions. J. Phys. Chem. B 115, 7175-7181 (2011).

34. Romano, F., Sanz, E. \& Sciortino, F. Crystallization of tetrahedral patchy particles in silico. J. Chem. Phys. 134, 174502 (2011).

35. Hamblin, G. D., Rahbani, J. F. \& Sleiman, H. F. Sequential growth of long DNA strands with user-defined patterns for nanostructures and scaffolds. Nature Commun. 6, 7065 (2015).

36. Alivisatos, A. P. et al. Organization of 'nanocrystal molecules' using DNA. Nature 382, 609-611 (1996).

37. Maye, M. M., Nykypanchuk, D., Cuisinier, M., van der Lelie, D. \& Gang, O. Stepwise surface encoding for high-throughput assembly of nanoclusters. Nature Mater. 8, 388-391 (2009).

38. Kim, J. W., Kim, J. H. \& Deaton, R. DNA-linked nanoparticle building blocks for programmable matter. Angew. Chem. Int. Ed. 50, 9185-9190 (2011).

39. Winfree, E., Liu, F., Wenzler, L. A. \& Seeman, N. C. Design and self-assembly of two-dimensional DNA crystals. Nature 394, 539-544 (1998).

40. Yan, H., Park, S. H., Finkelstein, G., Reif, J. H. \& LaBean, T. H. DNA-templated self-assembly of protein arrays and highly conductive nanowires. Science 301, 1882-1884 (2003).

41. He, Y., Chen, Y., Liu, H., Ribbe, A. E. \& Mao, C. Self-assembly of hexagonal DNA two-dimensional (2D) arrays. J. Am. Chem. Soc. 127, 12202-12203 (2005).
42. Liu, W., Zhong, H., Wang, R. \& Seeman, N. C. Crystalline two-dimensional DNA-origami arrays. Angew. Chem. Int. Ed. 50, 264-267 (2011).

43. Liu, Y., Ke, Y. \& Yan, H. Self-assembly of symmetric finite-size DNA nanoarrays. J. Am. Chem. Soc. 127, 17140-17141 (2005).

44. Zheng, J. et al. From molecular to macroscopic via the rational design of a selfassembled 3D DNA crystal. Nature 461, 74-77 (2009).

45. Rothemund, P. W. K. Folding DNA to create nanoscale shapes and patterns. Nature 440, 297-302 (2006).

46. Woo, S. \& Rothemund, P. W. K. Self-assembly of two-dimensional DNA origami lattices using cation-controlled surface diffusion. Nature Commun. 5, 4889 (2014).

47. Ke, Y., Ong, L. L., Shih, W. M. \& Yin, P. Three-dimensional structures selfassembled from DNA bricks. Science 338, 1177-1183 (2012).

48. Flory, P. J. Principles of Polymer Chemistry (Cornell Univ. Press, 1953).

49. Zhang, Y. G., Lu, F., Yager, K. G., van der Lelie, D. \& Gang, O. A general strategy for the DNA-mediated self-assembly of functional nanoparticles into heterogeneous systems. Nature Nanotech. 8, 865-872 (2013).

50. Zhang, C. et al. A general approach to DNA-programmable atom equivalents. Nature Mater. 12, 741-746 (2013).

51. Seeman, N. C. De novo design of sequences for nucleic acid structural engineering. J. Biomol. Struct. Dyn. 8, 573-581 (1990).

\section{Acknowledgements}

Research carried out at the Center for Functional Nanomaterials, Brookhaven National Laboratory, was supported by the US Department of Energy, Office of Basic Energy Sciences, under Contract No. DE-AC02-98CH10886.

\section{Author contributions}

W.L. and O.G. conceived and designed the experiments. W.L. performed the experiments. W.L., Y.T. and O.G. analysed the data. J.H. and A.V.T. contributed to the theoretical/ numerical analysis. W.L. and O.G. wrote the paper. O.G. supervised the projects. All the authors discussed the results and commented on the manuscript.

\section{Additional information}

Supplementary information is available in the online version of the paper. Reprints and permissions information is available online at www.nature.com/reprints. Correspondence and requests for materials should be addressed to O.G.

\section{Competing financial interests}

The authors declare no competing financial interests. 